\title{
Experimental Research of Reliability of Plant Stress State Detection by Laser-Induced Fluorescence Method
}

\author{
Yury Fedotov, Olga Bullo, Michael Belov, and Viktor Gorodnichev \\ Faculty of Radioelectronics and Laser Techniques, Department of Laser and Optoelectronics Systems, \\ Bauman Moscow State Technical University, 2-ya Baumanskaya Ulitsa, Moscow 105005, Russia
}

Correspondence should be addressed to Yury Fedotov; fed@bmstu.ru

Received 9 November 2015; Revised 31 January 2016; Accepted 11 February 2016

Academic Editor: Giulio Cerullo

Copyright (C) 2016 Yury Fedotov et al. This is an open access article distributed under the Creative Commons Attribution License, which permits unrestricted use, distribution, and reproduction in any medium, provided the original work is properly cited.

Experimental laboratory investigations of the laser-induced fluorescence spectra of watercress and lawn grass were conducted. The fluorescence spectra were excited by YAG:Nd laser emitting at $532 \mathrm{~nm}$. It was established that the influence of stress caused by mechanical damage, overwatering, and soil pollution is manifested in changes of the spectra shapes. The mean values and confidence intervals for the ratio of two fluorescence maxima near 685 and $740 \mathrm{~nm}$ were estimated. It is presented that the fluorescence ratio could be considered a reliable characteristic of plant stress state.

\section{Introduction}

Fluorescence analysis is a widely used high-sensitivity method that is applied in many scientific and technical fields. A viable application of the technique is the analysis of plant state [1-14]. External factors can cause plants stress and make their growth abnormal. Stress conditions are difficult to detect by visual observation during the early growth stages of a plant; however, the laser-induced fluorescence method is effective in the remote detection of plant stress state.

Chlorophyll is the basic fluorescent component of green leaf in the red and far-red regions. The fluorescence spectrum of a green leaf at room temperature exhibits two maxima in the red band $(680-690 \mathrm{~nm})$ and in the far-red band $(730-$ $740 \mathrm{~nm})[1,6,8,15]$. The fluorescence spectrum of a stressed plant is deformed in comparison with that of a plant in a nonstressed state. This effect is caused by disturbing the photosynthetic process of a plant under stressed conditions. The fluorescence spectrum depends on different factors such as excitation wavelength, type of stress factor, and plant species.

There are wide experimental data on the fluorescence spectra of various plant species, both stressed and nonstressed, excited at wavelength ranges of $266-635 \mathrm{~nm} \mathrm{[2,}$ $10,11,15,16]$. However, a number of points remain to be investigated. One such point is the reliability of the plant state detection based on the differences in fluorescence spectra of samples of a plant species, grown under identical conditions, except that some samples were stressed and the others were not.

In this paper, experimental results of the analysis of fluorescence spectra variation of different samples of a plant species in both normal and stressed states are presented.

\section{Materials and Methods}

2.1. Laboratory Setup Description. The fluorescence spectra were excited at a wavelength of $532 \mathrm{~nm}$. It is common to use lasers with wavelengths at 337, 335, and $532 \mathrm{~nm}$ for fluorescence excitation in experimental research. The laser source used in this study was selected because of the advantages offered by the solid-state YAG:Nd laser at the wavelength of $532 \mathrm{~nm}$ (for remote sensing equipment development), in comparison with both the nitrogen gas laser at the wavelength of $337 \mathrm{~nm}$ and the solid-state YAG:Nd laser at the wavelength of $355 \mathrm{~nm}$ (the third harmonic of the YAG laser has lower pulse intensity than its second harmonic).

The laboratory configuration used to measure fluorescence spectra is shown in Figure 1.

An EKSPLA NL210 solid-state YAG:Nd laser with diode pumping and frequency doubling was used as the source of 


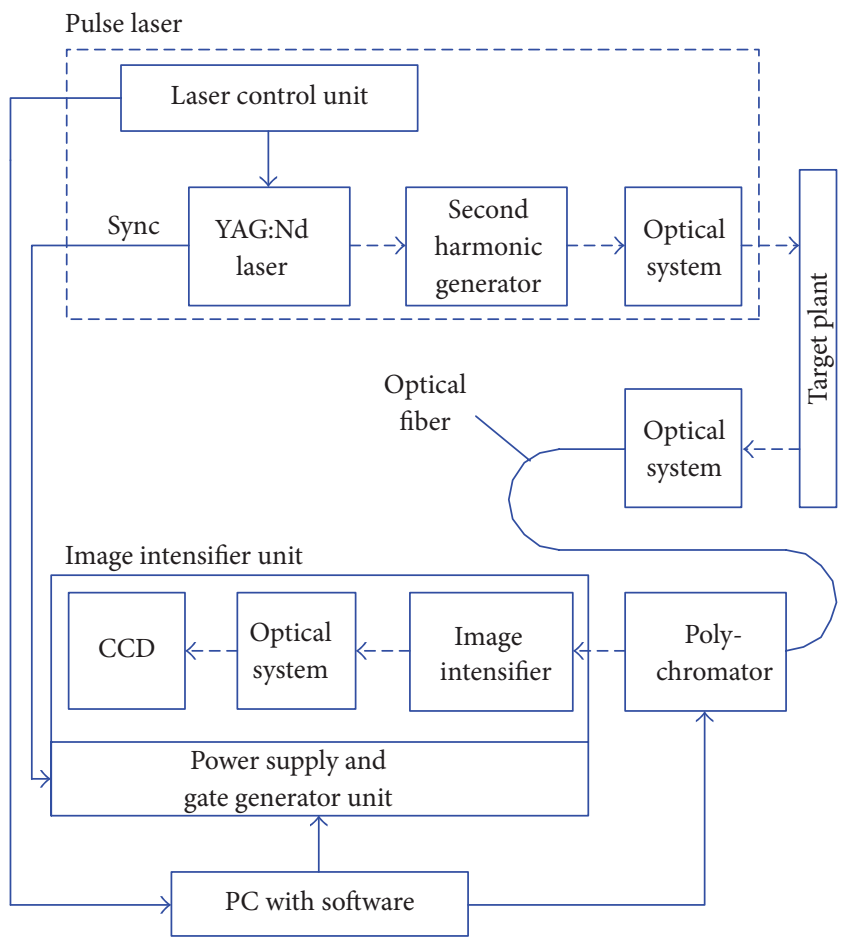

$-\rightarrow$ Optical signals

$\longrightarrow$ Electrical signals

FIGURE 1: Laboratory configuration for laser-induced fluorescence experiments.

fluorescence excitation. Laser light was transmitted by means of the optical system to the target plant located at a distance of $1 \mathrm{~m}$ from the optical system. The apparent diameter of the laser beam on the plant sample was approximately $25 \mathrm{~mm}$. The laser spot has covered 15-20 plants. The fluorescent radiation of the plants was collected from the same spot size together with the reflected laser light by the optical system and directed into the optical fiber. The optical fiber was used to transmit light to the input of the polychromator. The reflected light from the laser beam was prevented from entering a polychromator by using an NF01-532U Semrock filter. Fluorescent radiation from 595 to $800 \mathrm{~nm}$ was detected. An M266 Solar LS polychromator was used as the spectral device and all transitions within the polychromator fully automated (i.e., the swapping of diffraction grids and optical filters and slit width selection).

The fluorescence spectrum was detected using a highly sensitive detector (Matrix-430k-ns Deltatekh) based on CCD array with an image intensifier. The image intensifier (generation $\mathrm{II}+$, diameter $18 \mathrm{~mm}$ ) has quantum efficiency $15 \%$ at the wavelength $550 \mathrm{~nm}$. The image was transferred by the optical system from the image intensifier to the CCD. The image was converted into a digital array and transmitted to the computer. Special software developed with LabVIEW National Instruments was used to control the setup. The major specifications of the setup are presented in Table 1.

The experiment included equipment calibration as a preparatory step. The polychromator was calibrated by
TABLE 1: Specifications of laboratory setup.

\begin{tabular}{lc}
\hline Specifications & Value \\
\hline Laser pulse energy, mJ & 2.1 \\
Laser wavelength, nm & 532 \\
Laser pulse duration, ns & $<7$ \\
Laser repetition rate, Hz & $<500$ \\
Laser beam spread, mrad & $<3$ \\
Spectral band of registration, nm & $595-800$ \\
Spectral resolution, nm & 6 \\
Diameter of optical detection system, mm & 15 \\
Distance to sample, m & 1 \\
\hline
\end{tabular}

wavelength using a calibration light source based on a mercury-argon lamp (SL2 StellarNet Inc.) with a linear spectrum. The test was performed at the wavelength of $546.07 \mathrm{~nm}$. Calibration of sensitivity of the registration system was performed using a light source based on halogen lamp (DH2000-CAL Ocean Optics Inc.) with a continuous spectrum. Known spectrum of the lamp was acquired for sensitivity calculation.

2.2. Plant Samples. The experimental research of laserinduced fluorescence spectra was performed using easy to keep fast-growing plant species, that is, salads, watercress, mustard, common borage, cucumbers, and lawn grass. The experimental measurements of fluorescence spectra of watercress (Lepidium sativum) and lawn grass (that comprised a mixture of $30 \%$ perennial ryegrass (Lolium perenne), 65\% creeping red fescue (Festuca rubra), and 5\% sheep's ovina (Festuca ovina)) are presented in this paper. The research was conducted on plants in their normal state and under the influence of stress factors, for example, mechanical damage (leaf cutting and laying, root system damage), root system overwatering, and soil pollution (copper sulfate, $\mathrm{CuSO}_{4}$, ferric sulfate, $\mathrm{FeSO}_{4}$, and sodium chloride, $\mathrm{NaCl}$ ).

2.3. Normal and Stress Conditions. The plants in normal state were grown in favorable condition for their development. The watercress plants have height of approximately $4 \mathrm{~cm}$, and the lawn grass plants $8 \mathrm{~cm}$.

By the leaf cutting of the watercress, the half of one leaf of each plant was dissected. The leaf laying was conducted using $7 \times 7 \mathrm{~cm}$ flat plate with $200 \mathrm{~g}$ weight during approximately $1 \mathrm{~min}$. For root system damage in seedlings pots was cut a slit at the depth of $2 \mathrm{~cm}$, the root system has been damaged through the slit by means of utility knife, and then the slit was closed.

The overwatering stress condition was implemented by placing the pot of the watercress sample in a watering can. The level of water in the watering can was always slightly below the level of soil in the plant pot; thus, it was not visually obvious that the root system of the plant sample was constantly in overwatered soil. 


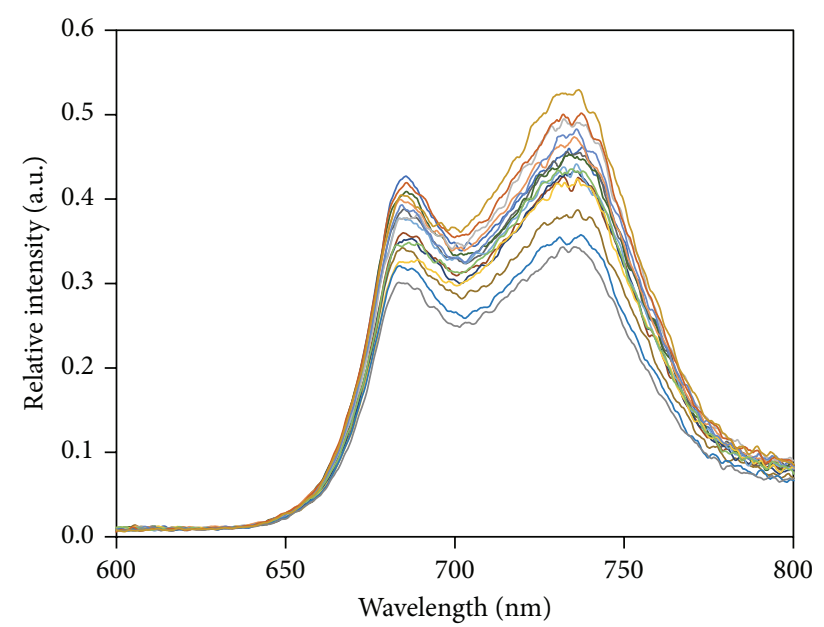

FIGURE 2: Fluorescence spectra of different watercress samples in normal state.

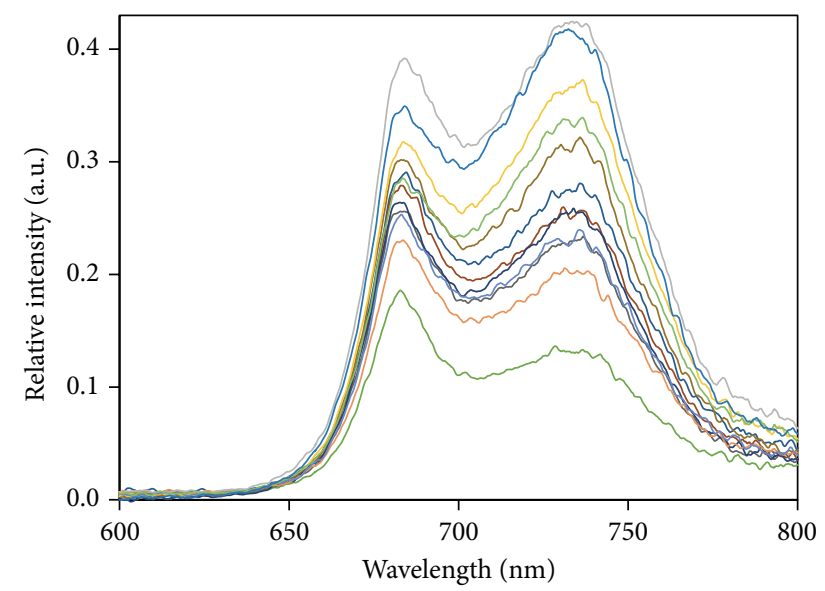

FigURE 3: Fluorescence spectra of different watercress samples in stressed state caused by leaf laying.

\section{Results and Discussion}

3.1. Fluorescence Spectra of Different Watercress Samples in Normal State. The fluorescence spectra of different samples of watercress grown under normal conditions are shown in Figure 2. The different plots in Figure 2 correspond to different plant samples that were planted at the same time and grown under the same conditions. The measurements were conducted in 16 days after planting.

As it can be seen in Figure 2, there are insignificant changes in the shapes of the fluorescence spectra from one sample to another, despite the differences in spectra intensity.

\subsection{Fluorescence Spectra of Watercress Stressed by Mechanical} Damage. The fluorescence spectra of different samples of watercress stressed by leaf laying mechanical damage are shown in Figure 3. There have been several experimental researches on the fluorescence spectra of plants in stressed states caused by different types of mechanical damage $[9,13$, 14], but few or none investigated the fluorescence spectra at an excitation wavelength of $532 \mathrm{~nm}$ [9].

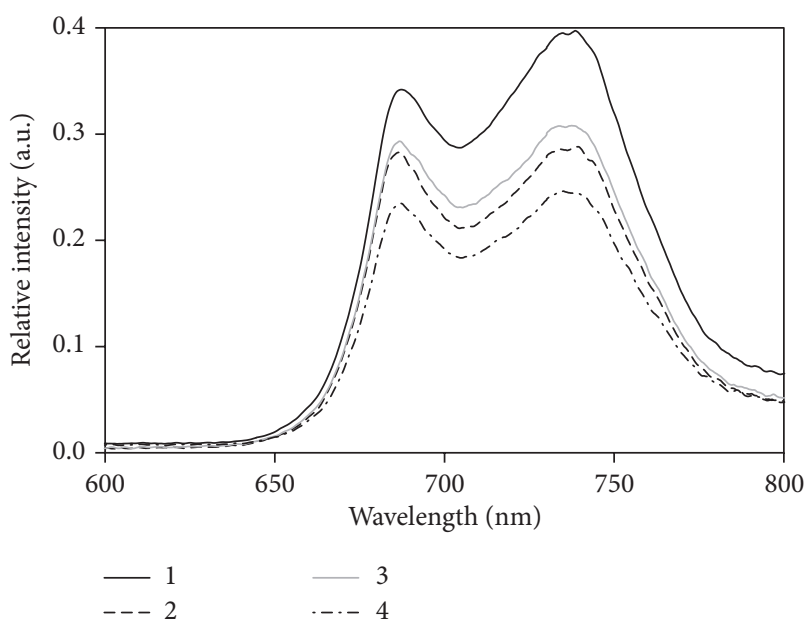

FIgURE 4: Averaged fluorescence spectra of watercress samples in normal and stressed conditions: (1) normal state, (2) leaf laying stress, (3) leaf cutting stress, and (4) root system damage stress.

The fluorescence spectra of watercress in a stressed state caused by leaf laying fluctuate considerably (Figure 3) and differ from those of watercress in a normal state (Figure 2). A similar difference is found between the fluorescence spectra of watercress in normal and stressed states when the stress is caused by mechanical damage of root system.

The differences between the fluorescence spectra of plants under normal and stressed conditions are illustrated clearly by averaging the measurements of the fluorescence spectra. Figure 4 displays the averaged fluorescence spectra of watercress in a normal state (plot 1) and stressed state by the mechanical damage of leaf laying (plot 2), leaf cutting (plot 3 ), and root system damage (plot 4). Plot 1 in Figure 4 corresponds to the averaged fluorescence spectra over the result of 20 measurements. Plots 2, 3, and 4 in Figure 4 correspond to the averaged fluorescence spectra over 11 measurements for each stress factor; thus, a single measurement corresponds to the measurement of a single fluorescence spectrum of a plant sample in definite time intervals from 20 to $40 \mathrm{~min}$ from the start of the stress factor influence.

It is clearly illustrated in Figure 4 that the shapes of the laser-induced fluorescence spectra of watercress in stressed conditions were caused by various types of mechanical damage change significantly. The ratios of fluorescence intensity in the red region $(680-690 \mathrm{~nm})$ and far-red region (730$740 \mathrm{~nm}$ ) increase in stress conditions.

\subsection{Fluorescence Spectra of Watercress in Stress State Caused} by Overwatering. The laser-induced fluorescence spectra of watercress in stressed state caused by overwatering are comparable with those presented in Figures 3 and 4 . Figure 5 shows the fluorescence spectra of watercress in stressed state caused by overwatering during 24 days (different spectra correspond to different measurements and plant samples).

As it is clearly illustrated in Figure 5 the fluorescence spectra of the watercress in a stressed state caused by overwatering during 24 days differ from those of watercress in a normal state. Furthermore, the spectra of the stressed samples 


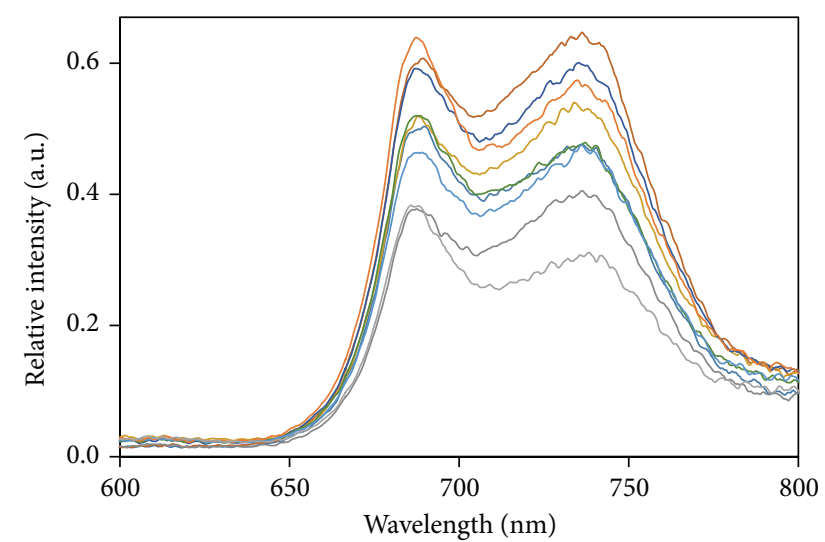

FIGURE 5: Fluorescence spectra of different watercress samples in stressed state caused by overwatering during 24 days.

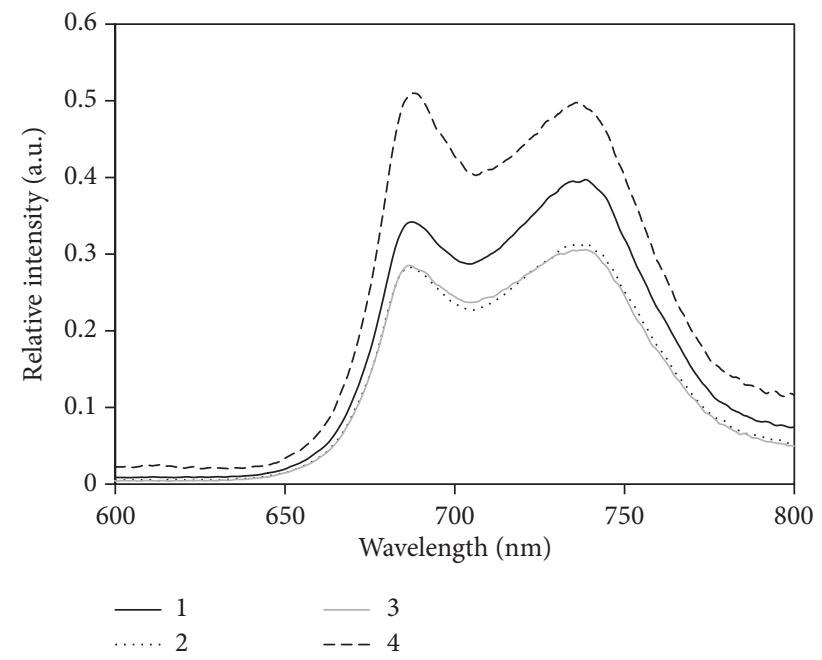

FIGURE 6: Fluorescence spectra of different watercress samples in stressed state caused by overwatering: (1) normal state, (2) 11-day overwatering, (3) 17-day overwatering, (4) 24-day overwatering.

fluctuate considerably, comparable with the fluorescence spectra of the watercress stressed by mechanical damage.

Figure 6 shows the laser-induced fluorescence spectra of watercress averaged over the number of plant samples and measurements (18 measurements for watercress in normal condition and 9 measurements for watercress in stressed condition).

Plot 1 in Figure 6 corresponds to the averaged fluorescence spectrum of watercress in a normal state. Plots 2, 3, and 4 in Figure 6 correspond to the averaged fluorescence spectra of watercress in stressed condition caused by overwatering during 11, 17, and 24 days, respectively. It can be clearly seen that the influence of the stress factor (overwatering in this case) accumulates gradually over the time of abnormal watering, increasing the fluorescence intensity.

The results presented in Figures 2-6 are in agreement with those of other experimental researches $[8,12]$ on plants under nitrogen stress and soil pollution using a fluorescence excitation source at the wavelength of $532 \mathrm{~nm}$.

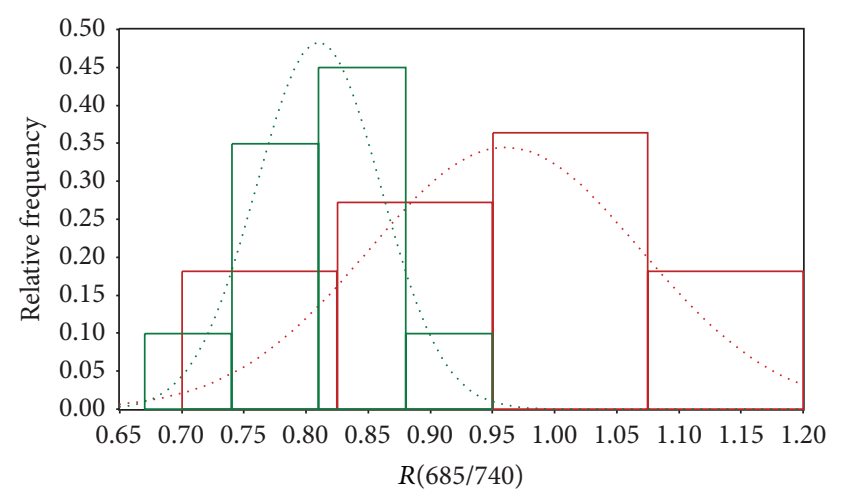

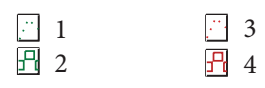

FIgURE 7: Histograms of distribution of fluorescence ratio, for watercress in a normal state and in a stressed state caused by leaf laying: (1) histogram for normal state, (2) histogram approximation for normal state, (3) histogram for stressed state, and (4) histogram approximation for stressed state.

3.4. Fluorescence Ratio. The ratio of fluorescence intensities in the 680-690 and 730-740 $\mathrm{nm}$ spectral bands is widely used in experimental research to characterize the fluorescence spectrum shape. Analysis of experimental data indicated that the ratio of fluorescence intensities near 685 and $735 \mathrm{~nm}$ can be used to characterize plant stress state.

Histograms of distribution of fluorescence intensities ratio $(R)$ at 685 and $740 \mathrm{~nm}$ in narrow spectral bands with bandwidths of $10 \mathrm{~nm}$, for watercress in a normal state in 16 days after planting and in a stressed state caused by leaf laying, are shown in Figure 7. Histograms were approximated by Gaussian function.

The mean value of the fluorescence ratio is 0.81 and the standard deviation is 0.05 for the watercress plants in a normal state. The mean value of the fluorescence ratio is 0.96 and the standard deviation is 0.11 for the watercress plants in a stressed state caused by leaf laying.

Histograms of distribution of the fluorescence intensities ratio at 685 and $740 \mathrm{~nm}$ for watercress in normal condition in 16 days after planting and in a stressed condition after 24 days of overwatering are shown in Figure 8.

The mean value of the fluorescence ratio is 0.81 and the standard deviation is 0.05 for watercress plants in a normal state. The mean value of the fluorescence ratio is 0.97 and the standard deviation is 0.07 for watercress in a stressed state caused by overwatering during 24 days.

As it is shown in Figures 7 and 8, it is possible to mistake, using a single measurement of fluorescent ratio $R$, defining whether a plant is under normal or stressed conditions because the distributions are overlapped. A far reliable method for defining the condition of a plant consists in using mean value of the fluorescence ratio, even in the case of small set of measurements.

The mean values (with $95 \%$ confidence intervals) of the experimental laser-induced fluorescence spectra of watercress under different stress conditions (leaf cutting, leaf 


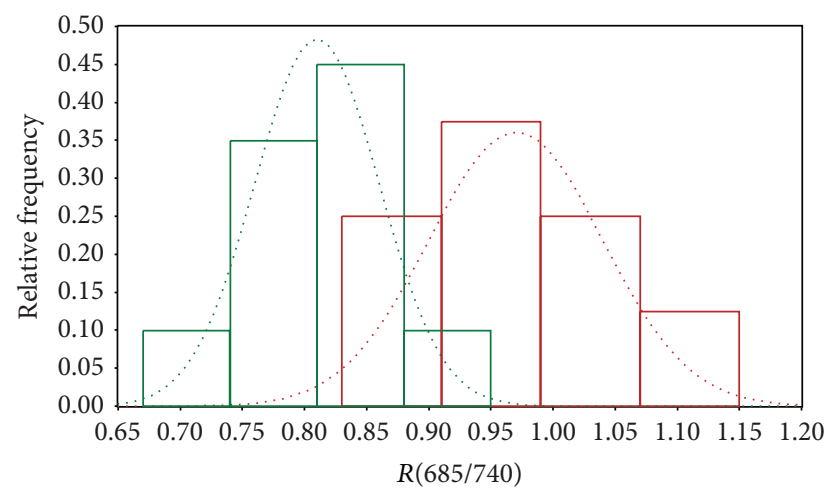

103

国 回 4

FIGURE 8: Histograms of distribution of fluorescence ratio, for watercress in a normal state and stressed state caused by overwatering for 24 days: (1) histogram for normal state, (2) histogram approximation for normal state, (3) histogram of stressed state, and (4) histogram approximation for stressed state.

laying, root system damage, and root system overwatering during 11, 17, and 24 days) are shown in Figure 9.

Columns 1, 3, 5, 7, 9, and 11 in Figure 9 correspond to the plants in a normal state and columns $2,4,6,8,10$, and 12 correspond to plants in a stressed state (2: leaf laying, 4: leaf cutting, 6: root system damage, 8: overwatering during 11 days, 10: overwatering during 17 days, and 12: overwatering during 24 days).

The changes of fluorescence spectra for plants in stress conditions described above are typical not only for watercress but also for other plants in stress conditions caused by different impact. The effect of soil pollution on lawn grass is considered below.

The aggregated statistical results (mean values and 95\% confidence intervals) of the experimental laser-induced fluorescence spectra of lawn grass under different stress conditions caused by soil pollution (copper sulfate, $\mathrm{CuSO}_{4}$, ferric sulfate, $\mathrm{FeSO}_{4}$, and sodium chloride, $\mathrm{NaCl}$ ) are shown in Figure 10.

Columns 1, 3, 5, 7, and 9 in Figure 10 correspond to lawn grass in a normal state (experimental research was conducted six weeks after planting, directly before the soil was polluted). Columns 2, 4, 6, 8, and 10 in Figure 10 correspond to lawn grass in a stressed state; measurements were performed 2 weeks after the initial influence of the stress factor for columns 2 and 6 and 4 weeks after the initial influence of the stress factor for columns 4,8 , and 10 . The stress factor was soil pollution by sodium chloride, $\mathrm{NaCl}$ ( 5 g per plant sample, columns 2 and 4), ferric sulfate, $\mathrm{FeSO}_{4}$ (3 g per plant sample, columns 6 and 8), and copper sulfate, $\mathrm{CuSO}_{4}(2 \mathrm{~g}$ per plant sample, column 10).

It is clearly illustrated in Figures 9 and 10 that the fluorescence ratio $(R)$ is characterized by stable and sufficient difference. The confidence intervals of the fluorescence ratio for plants under normal and stressed states were not large ( $\leq 0.1$ in the majority of the cases). The sum of confidence intervals of the fluorescence ratio $(R)$ for plants under normal and stressed states is not usually more than the difference

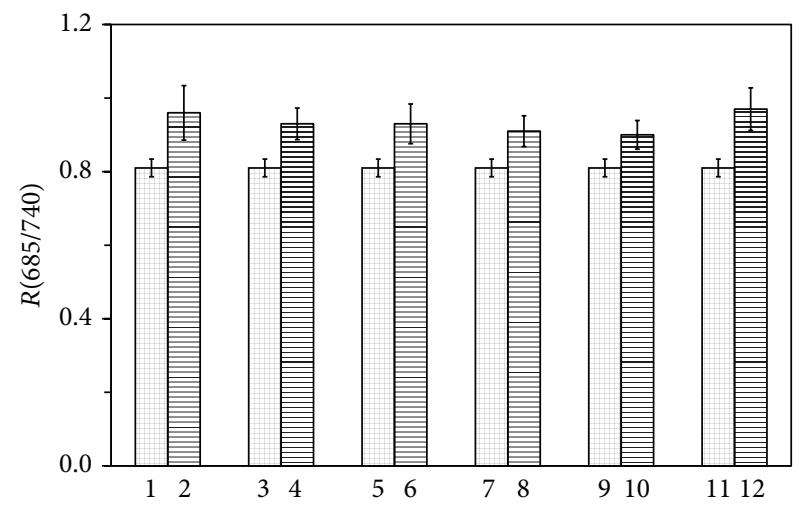

FIGURE 9: Fluorescence ratio $(R)$ mean values and $95 \%$ confidence intervals of watercress in normal conditions (columns 1, 3, 5, 7, and 9) and under stressed conditions caused by mechanical damage and overwatering (columns 2, 4, 6, 8, 10, and 12).

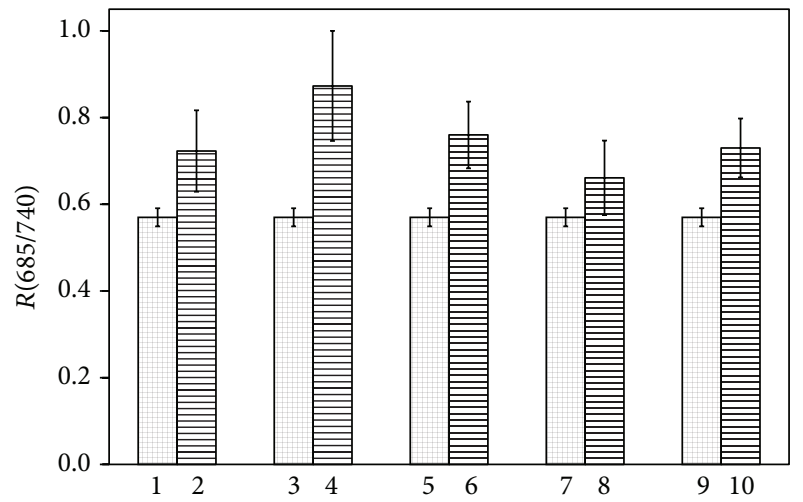

Figure 10: Fluorescence ratio $(R)$ mean values and 95\% confidence intervals of lawn grass under normal conditions (columns 1, 3, 5, 7 , and 9) and stressed conditions caused by different soil pollutants (columns 2, 4, 6, 8, and 10).

between ratio $R$ for plants in a normal state and ratio $R$ for plants in a stressed state caused by various factors (mechanical damage, overwatering, and soil polluting).

This means that fluorescence excitation at $532 \mathrm{~nm}$ wavelength and the ratio of fluorescence intensities in the red $(685 \mathrm{~nm})$ and far-red $(740 \mathrm{~nm})$ bands can be used as signatures of plant stress state caused by various factors.

\section{Conclusions}

By the processing of the experimental results of fluorescence spectra (induced by a $532 \mathrm{~nm}$ wavelength laser) of plants in normal and stressed states caused by mechanical damage, overwatering, and soil pollution the following conclusions can be postulated.

(i) The fluorescence spectra of different samples of a plant species revealed repeatability of the spectra shapes. Ratio $R$ of the fluorescence intensity at 685 and $740 \mathrm{~nm}$ demonstrated sufficient stability. However, it is possible to mistake defining the plant stress state (normal or stressed) using only single measurement of ratio $R$. We proposed more reliable method 
to define plant condition using mean value of $R$ ratio, which is suitable for small set of measurements.

(ii) The difference between the mean value of ratio $R$ for a plant in a normal state and that in a stressed state, in the majority of cases, is greater than the difference between ratio $R$ values for different samples of one plant species.

The experimental results obtained allow us to develop a remote laser system for detecting plant stress state. However, to ensure the reliability of the measurements, it is necessary to calculate the mean value of ratio $R$ for several measurements for several plants.

\section{Competing Interests}

The authors declare that they have no competing interests.

\section{References}

[1] A. S. Ndao, A. Konté, M. Biaye, M. E. Faye, N. A. B. Faye, and A. Wagué, "Analysis of chlorophyll fluorescence spectra in some tropical plants," Journal of Fluorescence, vol. 15, no. 2, pp. 123129, 2005.

[2] A. Takeuchi, Y. Saito, T. D. Kawahara, and A. Njmura, "Possibility of disease process monitoring of plants by laser-induced fluorescence method," in Proceedings of the Development and Evaluation of LIF Measurement Systems, vol. 4153 of Proceedings of SPIE, pp. 22-29, February 2001.

[3] Y. Saito, "Laser-induced fluorescence spectroscopy/technique as a tool for field monitoring of physiological status of living plants," in 14th International School on Quantum Electronics: Laser Physics and Applications, 66041W, vol. 6604 of Proceedings of SPIE, 12 pages, March 2007.

[4] H. A. Hristov, E. G. Borisova, L. A. Avramov, and I. N. Kolev, "Applications of laser-induced fluorescence for remote sensing," in Proceedings of the 11th International School on Quantum Electronics: Laser Physics and Applications, vol. 4397 of Proceedings of SPIE, pp. 496-500, Varna, Bulgaria, April 2001.

[5] K. J. Lee, Y. Park, A. Bunkin, R. Nunes, S. Pershin, and K. Voliak, "Helicopter-based lidar system for monitoring the upper ocean and terrain surface," Applied Optics, vol. 41, no. 3, pp. 401-406, 2002.

[6] L. A. Corp, J. E. McMurtrey, E. M. Middleton, C. L. Mulchi, E. W. Chappelle, and C. S. T. Daughtry, "Fluorescence sensing systems: in vivo detection of biophysical variations in field corn due to nitrogen supply," Remote Sensing of Environment, vol. 86, no. 4, pp. 470-479, 2003.

[7] M. V. Grishaev, V. V. Zuev, and O. V. Kharchenko, "Fluorescent channel of the Siberian Lidar Station," in 15th Symposium on High-Resolution Molecular Spectroscopy, 65800U, vol. 6580 of Proceedings of SPIE, 6 pages, December 2006.

[8] G. Matvienko, V. Timofeev, A. Grishin, and N. Fateyeva, "Fluorescence lidar method for remote monitoring of effects on vegetation," in Lidar Technologies, Techniques, and Measurements for Atmospheric Remote Sensing II, 63670F, vol. 6367 of Proceedings of SPIE, 8 pages, October 2006.

[9] J. Belasque, M. C. G. Gasparoto, and L. G. Marcassa, "Detection of mechanical and disease stresses in citrus plants by fluorescence spectroscopy," Applied Optics, vol. 47, no. 11, pp. 19221926, 2008.
[10] A. S. Gouveia-Neto, E. A. Silva, R. A. Oliveira et al., "Water deficit and salt stress diagnosis through LED induced chlorophyll fluorescence analysis in Jatropha curcas L. oil plants for biodisiel," in Imaging, Manipulation, and Analysis of Biomolecules, Cells, and Tissues IX, 79020A, vol. 7902 of Proceedings of SPIE, 10 pages, February 2011.

[11] R. Maurya, S. M. Prasad, and R. Gopal, "LIF technique offers the potential for the detection of cadmium-induced alteration in photosynthetic activities of Zea Mays L.," Journal of Photochemistry and Photobiology C: Photochemistry Reviews, vol. 9, no. 1, pp. 29-35, 2008.

[12] E. Middleton, J. E. McMurtrey, P. K. Entcheva Campbell, L. A. Corp, L. M. Butchera, and E. W. Chappellea, "Optical and fluorescence properties of corn leaves from different nitrogen regimes," in Remote Sensing for Agriculture, Ecosystems, and Hydrology IV, vol. 4879 of Proceedings of SPIE, pp. 72-83, March 2003.

[13] H. K. Lichtenthaler and U. Rinderle, "The role of chlorophyll fluorescence in the detection of stress conditions in plants," CRC Critical Reviews in Analytical Chemistry, vol. 19, no. 1, pp. 29-85, 1988.

[14] G. G. Matvienko, A. I. Grishin, O. V. Kharchenko, and O. A. Romanovskii, "Remote sounding of vegetation characteristics by laser-induced fluorescence," in Proceedings of the Laser Radar Technology and Applications IV, vol. 3707 of Proceedings of SPIE, pp. 524-532, Orlando, Fla, USA, 1999.

[15] Z. G. Cerovic, G. Samson, F. Morales, N. Tremblay, and I. Moya, "Ultraviolet-induced fluorescence for plant monitoring: present state and prospects," Agronomie, vol. 19, no. 7, pp. 543-578, 1999.

[16] A. B. Utkin, R. Felizardo, C. Gameiro, A. R. Matos, and P. Cartaxana, "Laser induced fluorescence technique for environmental applications," in Proceedings of the 2nd International Conference on Applications of Optics and Photonics, vol. 9286 of Proceedings of SPIE, Aveiro, Portugal, August 2014. 

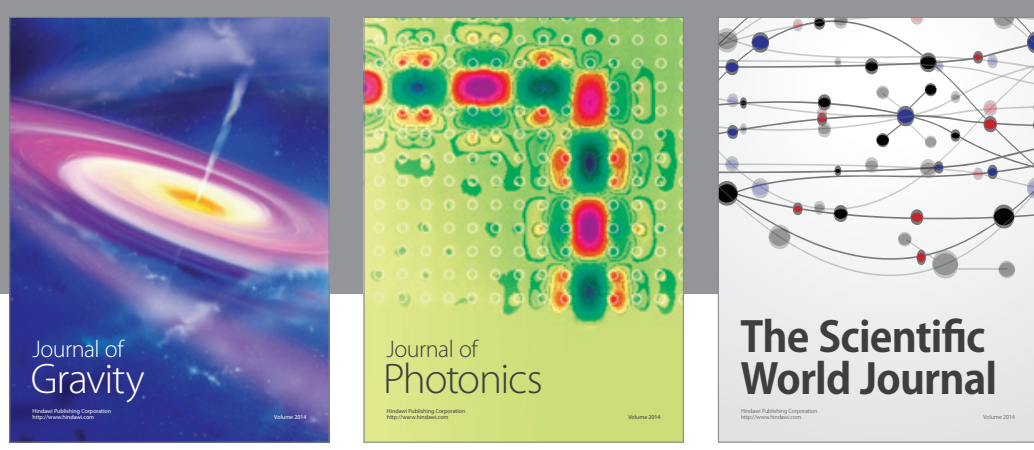

The Scientific World Journal
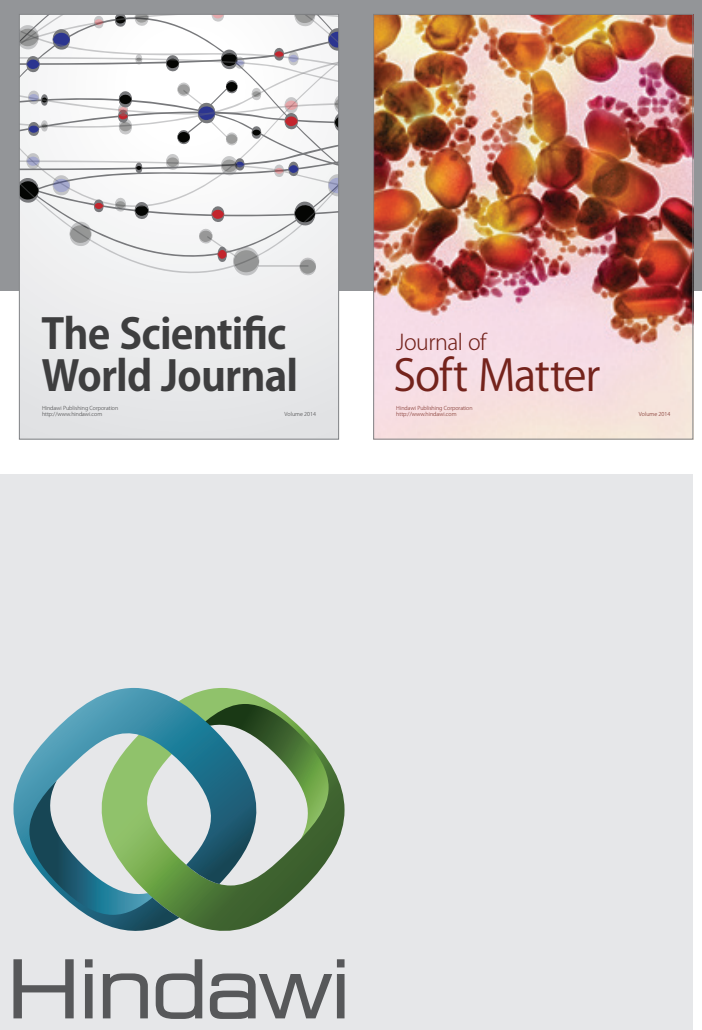

Submit your manuscripts at

http://www.hindawi.com

nternational Journal of

Statistical Mechanics
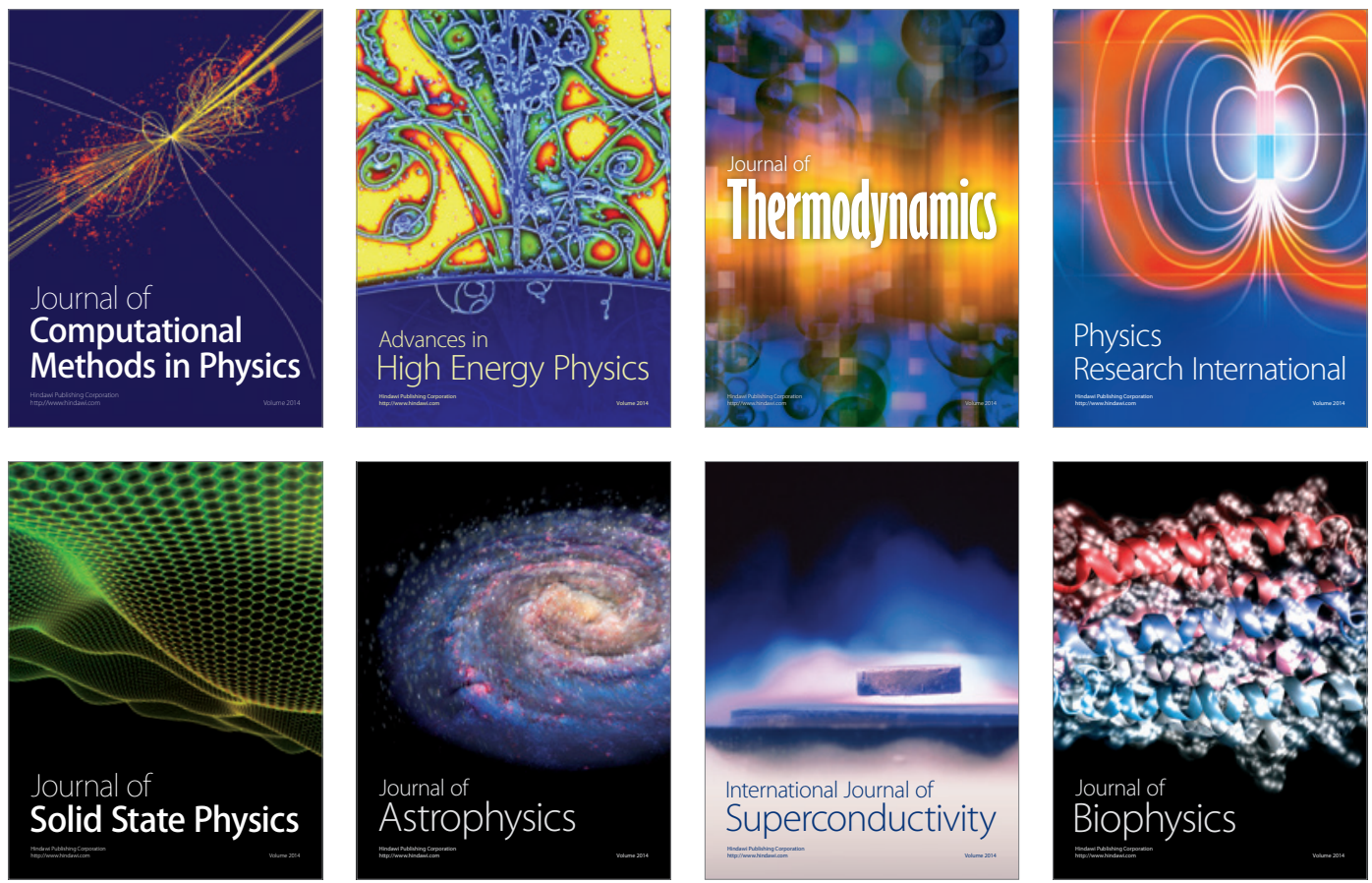
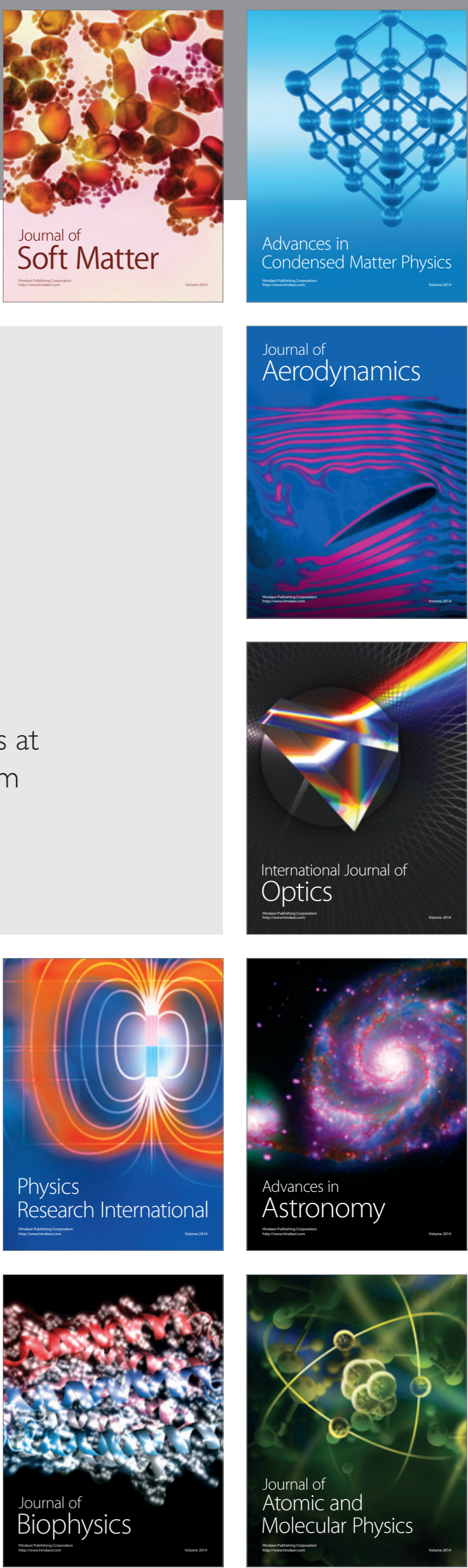